\title{
Family functioning and health-related quality of life in adolescents with pediatric inflammatory bowel disease
}

\author{
Michele Herzera, Lee A. Denson $^{a, b}$, Robert N. Baldassano ${ }^{c, d}$, and Kevin A. Hommel ${ }^{a, b}$ \\ aCincinnati Children's Hospital Medical Center \\ bUniversity of Cincinnati College of Medicine, Ohio \\ 'Children's Hospital of Philadelphia \\ dUniversity of Pennsylvania School of Medicine, Pennsylvania, USA
}

\begin{abstract}
Objectives-To examine the relationship between family functioning and health-related quality of life (HRQOL) in a sample of adolescents with inflammatory bowel disease (IBD), and to specify the domains of family functioning with which these families experience difficulties.

Methods-Sixty-two adolescents, aged 13-17 years, with a confirmed diagnosis of IBD completed assessments of HRQOL. Each adolescent's primary caregiver completed a measure of family functioning. Pediatric gastroenterologists provided data for disease severity assessments.
\end{abstract}

Results-A series of multivariate analyses of variance showed that adolescents from families with clinically elevated difficulties in problem solving, communication, and general family functioning endorsed lower HRQOL (i.e., social functioning, general well-being) after statistically controlling the effects of disease severity and diagnosis. As many as $25 \%$ of families reported clinically elevated difficulties across domains of family functioning.

Conclusions-Findings suggest that family functioning may be an important predictor of HRQOL among the adolescents with IBD, and that many families experience difficulties in their daily interactions. Close monitoring of family functioning may be a salient feature for prevention and intervention efforts and beneficial in promoting optimal psychosocial outcomes among the adolescents with IBD.

\section{Keywords}

adolescents; family functioning; inflammatory bowel disease; quality of life

\section{Introduction}

During childhood and adolescence, a diagnosis of inflammatory bowel disease (IBD) can impact health-related quality of life (HRQOL). Complex, rapidly changing, and timeconsuming treatments, combined with unpleasant, unpredictable, and potentially embarrassing symptoms (e.g., frequent trips to the bathroom, diarrhea) can all impose difficulties on the well-being and overall functioning of youth with IBD. In fact, earlier

\footnotetext{
(C) 2010 Wolters Kluwer Health | Lippincott Williams \& Wilkins.

Correspondence to: Kevin A. Hommel, PhD, Cincinnati Children's Hospital Medical Center, Division of Behavioral Medicine and Clinical Psychology, Center for Adherence and Self Management, 3333 Burnet Avenue MLC 7039, Cincinnati, OH 45229, USA, Tel: +513803 0407; fax: +513803 0415; kevin.hommel@cchmc.org.

Conflicts of interest: none declared.
} 
research has documented that children and adolescents with IBD report lower HRQOL in physical functioning, psychological functioning, and autonomy [1-3]. Given the value of patient-reported outcomes in health care, it is important for pediatric practitioners to focus not only on mortality and morbidity, but also on general well-being [4,5]. HRQOL provides a multidimensional view of an illness' impact on patients' lives and has become an important outcome variable for pediatric medical providers [6]. Indeed, recent research has shown a relationship between higher HRQOL and greater satisfaction with health care services among adolescents [7].

Among various pediatric chronic illness groups, several predictors of lower HRQOL have been identified. For example, among youth with epilepsy [8], diabetes [9], and obesity [10], depressive symptoms and psychosocial functioning have all been linked to lower HRQOL. Further, recent data among youth with end-stage renal disease [11] and diabetes [12] show a significant relationship between family functioning and HRQOL. Hence, psychosocial factors, including family functioning, have been related to HRQOL in some pediatric populations. However, pediatric IBD represents a considerably under-studied and underserved population with respect to psychosocial issues. Indeed, research on predictors of HRQOL among children and adolescents with IBD is limited to disease severity and, more recently, parent distress [13]. Although such individual-level variables are essential for understanding the factors that may impede HRQOL in youth with IBD, these do not capture the larger family context within which children and adolescents with IBD function. Moreover, the unique socially stigmatizing and limiting nature of pediatric IBD suggests that a better understanding of these patients' primary social support resource (i.e. family members) may be critical to maintaining optimal HRQOL. A recent review [14] of broad pediatric research shows that family functioning is a powerful determinant of overall functioning and well-being in youth with chronic medical conditions. Adaptive family relationships have been linked to positive psychological functioning $[9,15]$, whereas disruptions in family life have been linked to poorer emotional and behavioral functioning [16] and poor adherence to treatment regimens [17]. Among youth with IBD, family functioning has similarly been linked with psychosocial and health outcomes. For example, Tojek et al. [18] found positive associations between general family dysfunction, pain and fatigue, and the frequency of bowel movements among youth with IBD. Others have similarly shown that a family environment characterized by cohesion and open communication is a protective factor for the psychosocial functioning of youth with IBD, even in the presence of greater disease severity [19]. In contrast, greater family conflict is associated with poorer emotional functioning (e.g., greater depressive symptoms) [20]. Research also indicates that families with a child diagnosed with IBD may experience greater levels of family dysfunction compared with other chronic illness groups (e.g., diabetes) and healthy comparisons [19]. Poorer family communication has also been reported by parents of adolescents with IBD compared with healthy adolescents [21]. Yet, it remains unknown what proportion of families experience clinically significant levels of dysfunction across various domains of family functioning. Further, because the psychosocial factors that impact patient HRQOL vary across disease groups as a function of unique demands and aspects of specific conditions (e.g., onset, treatment course, and prognosis) [22], and there is a dearth of empirical evidence on HRQOL predictors in pediatric IBD, it is unclear to what extent family functioning and HRQOL are related in this unique population.

HRQOL among children and adolescents with IBD is an important clinical outcome variable that requires better understanding. However, few risk factors that contribute to low HRQOL in this population have been identified. Family functioning represents a salient and clinically relevant variable in IBD, which may be linked to youth's HRQOL. However, the nature and extent of family dysfunction, and its association with disease-specific quality of life in youth with IBD is unknown. Moreover, earlier research in IBD has underused IBD-specific 
HRQOL as an outcome, and no research on family functioning in IBD has been conducted using a measure of family functioning that is not only psychometrically sound [23], but also designed to assess family functioning in a multidimensional manner and be of maximal clinical utility $[24,25]$. This study was designed to (i) examine the relationship between various domains of family functioning and IBD-specific quality of life and (ii) assess the clinical significance of family dysfunction in this population. We hypothesized that youth from families with clinically elevated difficulties in family functioning would report poorer IBD-specific HRQOL.

\section{Materials and methods}

\section{Participants}

This study is a part of a larger longitudinal study, examining adherence to medications in adolescents with IBD. Participants included were adolescents aged 13-17 years with a confirmed diagnosis of IBD and a prescribed treatment regimen of 5-aminosalycylic acid and/or 6-MP/ azathioprine. Each adolescent's primary caregiver also participated in the study. Exclusion criteria included patients with a diagnosis of a neurocognitive disorder, comorbid chronic illness diagnosis, and lack of English fluency. Of the 83 patients contacted for recruitment, 13 declined participation, and eight did not provide complete data. Primary reasons for declining the participation included blood draw requirement (for adherence data as part of the larger study), time constraints, and/or lack of interest. Thus, a final sample of 62 adolescents ( 35 male, 27 female) receiving treatment for IBD (Crohn's disease $n=49$; ulcerative colitis $n=13$ ) and their caregivers participated in this study. The majority of adolescents was Caucasian (88.7\%) and had a mean age of 15.5 years [standard deviation $(\mathrm{SD})=1.4]$. Patient and caregiver characteristics are outlined in Table 1. Sample characteristics were generally representative of the pediatric IBD population and recently published studies in IBD [26].

\section{Procedure}

Study recruitment occurred at two large pediatric medical centers in the Midwest $(n=28)$ and the Northeast ( $n=34$ ) USA. Eligibility was determined by study personnel through chart review. Eligible families were contacted during regularly scheduled gastroenterology clinic appointments, scheduled infliximab infusions, or through telephone. Informed consent/ assent were obtained from both the adolescents and their caregivers, after first confirming the study eligibility with caregivers. Disease-severity assessments were completed by study personnel using data provided by each participant's gastroenterologist for the clinic appointment corresponding to the study visit or the most recent clinic appointment. Participants were compensated with $\$ 25$ for participation. Institutional review boards at each hospital approved this study.

\section{Measures}

Demographic questionnaire-A demographic questionnaire assessing the family characteristics (e.g., household income, marital status, parental education) was completed by parents.

Pediatric Crohn's disease activity index-The well-validated pediatric Crohn's disease activity index [27] assesses Crohn's disease activity using both the subjective (e.g., pain) and the objective criteria (e.g., physical exam), laboratory findings, and growth parameters. Scores range from 0-100: less than 10=inactive disease; $10-29=$ mild disease, and more than or equal to $30=$ moderate-to-severe disease activity [28]. Internal consistency was 0.95 in this sample. 
Lichtiger colitis activity index-The Lichtiger colitis activity index (LCAI) [29] uses both the subjective and the objective criteria to assess eight ulcerative colitis symptoms (score 0-21): daily stool frequency, nocturnal diarrhea, visible blood in stool, fecal incontinence, abdominal pain or cramping, general well-being, abdominal tenderness, and need for antidiarrheal medication, with higher scores representing more severe disease. LCAI scores range from $0-21$. Scores less than or equal to 2 indicate quiescent disease; less than 10 indicate a response to therapy; more than or equal to 10 indicate active disease and no response to therapy [30]. Internal consistency was 0.85 in this sample.

Family assessment device-The family assessment device (FAD) [25] is a 60-item measure that assesses family functioning on six different dimensions: problem solving (ability to resolve problems), communication (exchange of clear and direct verbal information), roles (division of responsibility for completing family tasks), affective responsiveness (ability to respond with appropriate emotion), affective involvement (degree to which family members are involved and interested in one another), and behavior control (manner used to express and maintain standards of behavior). The FAD also includes an independent dimension of general functioning (overall functioning of family). For each FAD item, individuals rate their level of agreement/disagreement on specific family behaviors (e.g., 'We try to think of different ways to solve problems' and 'We don't talk to each when we are angry') using a 4-point Likert scale ranging from 1 (strongly agree) to 4 (strongly disagree). Higher scores are indicative of poorer family functioning. Clinical cutoff scores differentiating 'healthy' (i.e., nonclinical) versus 'unhealthy' (i.e., clinical) family functioning for each dimension have been documented [24]. Clinical cutoff scores are as follows: problem solving $=2.20$; communication $=2.20$; roles $=2.30$; affective responsiveness $=2.20$; affective involvement $=2.10$; behavior control $=1.90$; general functioning $=2.00$. For this study, internal consistencies for FAD dimensions were as follows: problem solving, $a=0.72$; communication, $a=0.82$; roles, $a=0.68$; affective responsiveness, $a=0.80$; affective involvement, $a=0.75$; behavior control, $a=0.65$; general functioning, $a=0.85$. Parents completed the FAD in this sample.

IMPACT-III-The IMPACT-III [31] is a 35-item patient self-report, IBD-specific measure of HRQOL. It assesses the extent to which an adolescent is affected by a particular issue (e.g., stomach pain, missing out on certain activities) using 5-point Likert scaling, with lower scores indicating poorer HRQOL. Originally, six domains (i.e., bowel symptoms, systemic symptoms, social/functional concerns, body image, test and treatment concerns, and emotional concerns) were proposed. However, a recent examination of this measure's factor structure showed 4 factors with good-to-excellent reliability: general well-being, emotional functioning, social functioning, and body image [32]. This factor structure was used in this study, along with a total score of HRQOL created by adding all the 35 items. This total score showed excellent internal consistency for the current sample (Cronbach's $a=0.95$ ).

\section{Statistical analyses}

The pediatric Crohn's disease activity index and the LCAI scores were calculated as continuous measures and combined to form one continuous measure of disease severity for the primary analyses. Descriptive statistics including means and SDs were calculated for all the variables. Next, $t$ tests and correlation analyses between sociodemographic/medical characteristics and IMPACT-III scores were done to identify potential covariates. FAD scores for each dimension were then dichotomized as 'clinical' and 'nonclinical' according to cutoffs established by the researchers of the FAD [24]. Next, a series of multivariate analysis of variance tests were carried out to examine significant group differences (i.e., between 'clinical' and 'nonclinical' family functioning) on IMPACT-III scores (i.e., general 
well-being, emotional functioning, social functioning, body image, and total HRQOL). Lastly, the percentage of families meeting clinical cutoff scores for each FAD dimension was calculated. Analyses were done in SPSS 15.0.1 (SPSS Inc., Chicago, Illinois, USA).

\section{Results \\ Descriptive statistics}

The means and the SDs for main study variables are presented in Table 1. Significant correlations were found between the disease severity and all IMPACT-III scores: general well-being, $r=-0.47, P$ value of less than 0.001 ; emotional functioning, $r=-0.39, P$ value of less than 0.01 ; social functioning, $r=-0.27, P$ value of less than 0.05 ; body image, $r=-0.32$, $P=0.01$; total HRQOL, $r=-0.45, P$ value of less than 0.001 . Significant differences in general well-being [ $t(59)=-2.91, P<0.05]$, emotional functioning [ $t(59)=-1.29, P<0.05]$, and total HRQOL $[t(59)=-2.23, P<0.01]$ were also found for IBD diagnosis; a diagnosis of Crohn's disease was associated with lower HRQOL ratings.

\section{Group differences on health-related quality of life}

After statistically controlling for variance in HRQOL contributed by disease severity and diagnosis by covarying these variables, a multivariate analysis of variance showed that youth from families reporting clinically elevated difficulties with problem solving reported lower social functioning, $F(60)=5.68, P$ value of less than 0.05 , than those from families in the nonclinical range. Patients from families reporting clinically elevated difficulties with communication reported lower general well-being, $F(59)=6.36, P$ value of less than 0.05 , and social functioning, $F(59)=12.68, P$ value of less than 0.01 . Lastly, youth from families reporting clinically elevated difficulties in general family functioning reported lower general well-being, $F(59)=4.22, P$ value of less than 0.05 , and social functioning, $F(60)=6.46, P$ value of less than 0.05 .

\section{Proportion of families meeting clinical cutoffs of family functioning}

Overall, compared with pre-established cutoff scores for clinically elevated functioning, sample means on all FAD dimensions fell in the nonclinical range. However, a high percentage of families endorsed clinically elevated difficulties in functioning: $7.5 \%$ in problem solving, $18.8 \%$ in communication, $17.5 \%$ in roles, $12.5 \%$ in affective responsiveness, $25 \%$ in affective involvement, $7.5 \%$ in behavior control, and $16.3 \%$ in general functioning.

\section{Discussion}

This is the first study to examine family functioning as a potential predictor of diseasespecific HRQOL among adolescents with IBD. Few predictors of HRQOL in pediatric IBD have been identified, and these have predominantly been limited to disease characteristics, such as disease severity. Our findings are consistent with research indicating that greater disease severity [33] and a diagnosis of Crohn's disease (rather than ulcerative colitis) [2] are associated with lower HRQOL. However, these findings extend the IBD literature and provide support for emerging research in other pediatric populations (i.e., end-stage renal disease and diabetes) showing a link between family functioning and HRQOL [11,12]. Indeed, our results indicate that even after controlling for disease severity and IBD diagnosis, adolescents living in families who experience clinically elevated difficulties in several dimensions of family functioning experience significantly poorer IBD-specific HRQOL. 
Specifically, adolescents with IBD experienced significantly poorer HRQOL in general well-being and social functioning when their families endorsed clinically elevated dysfunction in areas of problem solving, communication, and general family functioning. To our knowledge, these family-related factors have never before been examined in pediatric IBD, nor examined as predictors to IBD-specific HRQOL. These findings might suggest that poor or inefficient problem solving and communication among these families may contribute to greater strain or conflict between family members or perhaps contribute to youth with IBD feeling unsupported by family members. Consequently, this may play a role in the development of poorer quality of life and functioning across several domains. This relationship is supported by recent research indicating that greater family conflict is associated with poorer HRQOL among youth with various medical [12,34] conditions. Our findings suggest that family functioning may also be a salient factor to consider when evaluating the HRQOL in adolescents with IBD.

Although sample means for all family functioning dimensions were in the nonclinical range, a substantial proportion of families endorsed clinically elevated family dysfunction. For example, $25 \%$ of families endorsed clinically elevated dysfunction in affective involvement, or the degree to which family members are involved and interested in one another. Elevations in affective involvement might indicate that certain family members are becoming overprotective of others, which may result in increased dependency on one another. In this case, it might suggest that parents would be overprotective of their child with IBD; however, this is an empirical question that would need to be investigated. Approximately, $19 \%$ of families perceived clinically elevated difficulties in communication among family members. These findings are consistent with earlier research, which documents less cohesion and poor communication among families with a chronically ill child $[21,35]$. In addition, approximately, $18 \%$ of families endorsed clinically elevated dysfunction with respect to the division of responsibility for completing family roles. These findings may reflect the natural progression and course of IBD (i.e., alternating periods of remission and flare-ups/medical crises) and the consequent impact on family life. IBD demands may also limit the degree to which families are involved and express interest in one another's lives and activities (i.e., affective involvement) and place constraints on families' ability to communicate effectively with one another. Family roles and responsibilities may also change to accommodate any flare-ups or exacerbations in symptoms. For example, in the event of an IBD-related hospitalization, caregiver roles may shift so that one can remain with the ill adolescent in the hospital while the other caregiver negotiates care for other children and assumes household tasks.

Our findings must be interpreted within the context of a few methodological limitations and implications for future research. First, disease characteristics of this sample (i.e., adolescents, predominantly Crohn's disease diagnosis and inactive disease status) may not generalize well to other, more diverse IBD samples with greater disease severity. The sociodemographics of this sample (i.e., Caucasians, mostly married and employed caregivers) may also limit generalizability; however, sample characteristics are comparable with other published studies of pediatric IBD [36]. Second, the descriptive, cross-sectional nature of data analyses precluded any examination of the causal links between family functioning and HRQOL or variations in these variables over time. As child and family functioning are interrelated and families are constantly changing to adapt to a child's chronic condition [37], longitudinal research is needed to more thoroughly examine family functioning and youth HRQOL over time. Moreover, although we minimized the number of statistical comparisons by using the modified factor structure of the IMPACT III (four subscales rather than six subscales as originally proposed), the use of the FAD and IMPACT III necessitated multiple comparisons, as there are no total scores for each measure. Thus, these findings should be viewed as preliminary, and future research should examine the 
relationship between these constructs in a large sample. Third, this study examined generic family functioning, not disease-specific features of family functioning that may be more salient. Yet to date, no IBD-specific measure of family functioning exists. Pediatric research that documents the advantages of examining disease-specific family functioning [34] substantiates the need for the development of an IBD-specific measure of this construct. In addition, as the self-report nature of the FAD may predispose families to overestimate their functioning, future research that incorporates observational measures of family functioning may provide an objective assessment of a family's actual behavior.

Given the push for pediatric providers to pay particular attention to patient-reported outcomes, such as HRQOL, greater knowledge of factors that may impact HRQOL among youth with IBD can substantially guide clinical interventions and practice. For example, the finding that up to $25 \%$ of families endorsed difficulties across family functioning dimensions highlights the importance of monitoring family functioning during routine clinic visits. This may identify families in need of support services or psychological intervention, and adolescents who may be at heightened risk of experiencing impaired HRQOL. Teaching effective communication and providing family guidance on the division of responsibilities for completing family roles and how to adaptively remain involved in each others' lives in the midst of IBD disease management, may prove particularly beneficial for parents and adolescents alike. In fact, family-based interventions that target communication and problem solving among families with chronically ill youth show great promise for improving health and psychosocial outcomes among these youth [38].

\section{Acknowledgments}

This study was supported in part by NIDDK K23 DK079037, PHS Grant P30 DK 078392, Procter and Gamble Pharmaceuticals, Prometheus Laboratories, Inc., and Institutional Clinical and Translational Science Award NIH/ NCRR Grant Number 1UL1RR026314.

\section{References}

1. De Boer M, Grootenhuis M, Derkx B, Last B. Health-related quality of life and psychosocial functioning of adolescents with inflammatory bowel disease. Inflamm Bowel Dis. 2005; 11:400406. [PubMed: 15803032]

2. Loonen HJ, Grootenhuis MA, Last BF, Koopman HM, Derkx HHF. Quality of life in paediatric inflammatory bowel disease measured by a generic and a disease-specific questionnaire. Acta Paediatr. 2002; 91:348-354. [PubMed: 12022311]

3. Cunningham C, Drotar D, Palermo TM, McGowan K, Arendt R. Health-related quality of life in children and adolescents with inflammatory bowel disease. Child Health Care. 2007; 6:29-43.

4. Sullivan M. The new subjective medicine: taking the patient's point of view on healthcare and health. Soc Sci Med. 2003; 56:1595-1604. [PubMed: 12614708]

5. Schor EL. The future pediatrician: promoting children's health and development. J Pediatr. 2007; 151 (5 Suppl):S11-S16. [PubMed: 17950316]

6. Hommel KA, Davis CM, Baldassano RN. Medication adherence and quality of life in pediatric inflammatory bowel disease. J Pediatr Psychol. 2008; 33:867-847. [PubMed: 18337262]

7. Mah JK, Tough S, Fung T, Douglas-England K, Verhoef M. Adolescent quality of life and satisfaction with care. J Adolesc Health. 2006; 38:607.e601-607.e607. [PubMed: 16635774]

8. Orrin D, Lauren W, Joyce C, Marc G, Kenneth P, Carol C. Risk factors for poor health-related quality of life in adolescents with epilepsy. Epilepsia. 1999; 40:1715-1720. [PubMed: 10612334]

9. Grey M, Boland EA, Yu C, Sullivan-Bolyai S, Tamborlane WV. Personal and family factors associated with quality of life in adolescents with diabetes. Diabetes Care. 1998; 21:909-914. [PubMed: 9614606]

10. Zeller MH, Modi AC. Predictors of health-related quality of life in obese youth. Obesity (Silver Spring). 2006; 14:122-130. [PubMed: 16493130] 
11. Goldstein SL, Graham N, Warady BA, Seikaly M, McDonald R, Burwinkle TM, et al. Measuring health-related quality of life in children with ESRD: performance of the generic and ESRDspecific instrument of the Pediatric Quality of Life Inventory (PedsQL). Am J Kidney Dis. 2008; 51:285-297. [PubMed: 18215706]

12. Weissberg-Benchell J, Nansel T, Holmbeck G, Chen R, Anderson B, Wysocki T, Laffel L. Steering Committee of the Family Management of Diabetes Study. Generic and diabetes-specific parent-child behaviors and quality of life among youth with type 1 diabetes. J Pediatr Psychol. 2009; 34:977-988. [PubMed: 19270028]

13. Herzer M, Denson LA, Baldassano RN, Hommel KA. Patient and parent psychosocial factors associated with health-related quality of life in pediatric inflammatory bowel disease. J Pediatr Gastroenterol Nutr. (in press).

14. Kazak, AE.; Rourke, MT.; Navsaria, N. Families and other systems in pediatric psychology. In: Roberts, MC.; Steele, RG., editors. Handbook of pediatric psychology. 4. New York City: The Guilford Press; 2009. p. 656-671.

15. Hanson CL, De Guire MJ, Schinkel AM, Henggeler SW, Burghen GA. Comparing social learning and family systems correlates of adaptation in youths with IDDM. J Pediatr Psychol. 1992; 17:555-572. [PubMed: 1432481]

16. Whittemore R, Kanner S, Singleton S, Hamrin V, Chiu J, Grey M. Correlates of depressive symptoms in adolescents with type 1 diabetes. Pediatr Diabetes. 2002; 3:135-143. [PubMed: 15016153]

17. Duke DC, Geffken GR, Lewin AB, Williams LB, Storch EA, Silverstein JH. Glycemic control in youth with type 1 diabetes: family predictors and mediators. J Pediatr Psychol. 2008; 33:719-727. [PubMed: 18296726]

18. Tojek TM, Lumley MA, Corlis M, Ondersma S, Tolia V. Maternal correlates of health status in adolescents with inflammatory bowel disease. J Psychosom Res. 2002; 52:173-179. [PubMed: 11897236]

19. Engstrom I. Inflammatory bowel disease in children and adolescents: mental health and family functioning. J Pediatr Gastroenterol Nutr. 1999; 28:S28-S33. [PubMed: 10204521]

20. Burke P, Kocoshis S, Chandra R, Whiteway M, Sauer J. Determinants of depression in recent onset pediatric inflammatory bowel disease. J Am Acad Child Adolesc Psychiatry. 1990; 29:608-610. [PubMed: 2387796]

21. Mackner LM, Crandall WV. Brief report: psychosocial adjustment in adolescents with inflammatory bowel disease. J Pediatr Psychol. 2006; 31:281-285. [PubMed: 15802606]

22. Rolland JS. Toward a psychosocial typology of chronic and life threatening illness. Fam Syst Med. $1984 ; 2: 245-262$.

23. Alderfer MA, Fiese BH, Gold JI, Cutuli JJ, Holmbeck GN, Goldbeck L, et al. Evidence-based assessment in pediatric psychology: family measures. J Pediatr Psychol. 2008; 33:1046-1061. [PubMed: 17905801]

24. Miller IW, Epstein NB, Bishop DS, Keitner GI. The mcmaster family assessment device: reliability and validity. J Marital Fam Ther. 1985; 11:345-356.

25. Miller IW, Kabacoff RI, Epstein NB, Bishop DS, Keitner GI, Baldwin LM, van der Spuy HIJ. The development of a clinical rating scale for the McMaster model of family functioning. Fam Process. 1994; 33:53-69. [PubMed: 8039568]

26. Mackner LM, Crandall WV. Oral medication adherence in pediatric inflammatory bowel disease. Inflamm Bowel Dis. 2005; 11:1006-1012. [PubMed: 16239847]

27. Hyams JS, Ferry GD, Mandel FS, Gryboski JD, Kibort PM, Kirschner BS, et al. Development and validation of a pediatric Crohn's disease activity index. J Pediatr Gastroenterol Nutr. 1991; 12:439-447. [PubMed: 1678008]

28. Hyams J, Markowitz J, Otley A, Rosh J, Mack D, Bousvaros A, et al. Evaluation of the pediatric Crohn disease activity index: a prospective multicenter experience. J Pediatr Gastroenterol Nutr. 2005; 41:416-421. [PubMed: 16205508]

29. Lichtiger S, Present DH, Kornbluth A, Gelernt I, Bauer J, Galler G, et al. Cyclosporine in severe ulcerative colitis refractory to steroid therapy. N Engl J Med. 1994; 330:1841-1845. [PubMed: 8196726] 
30. Fanjiang G, Russell GH, Katz AJ. Short- and long-term response to and weaning from infliximab therapy in pediatric ulcerative colitis. J Pediatr Gastroenterol Nutr. 2007; 44:312-317. [PubMed: 17325550]

31. Otley A, Smith C, Nicholas D, Avolio J, Sherman PM, Griffiths AM. The IMPACT questionnaire: a valid measure of health-related quality of life in pediatric inflammatory bowel disease. J Pediatr Gastroenterol Nutr. 2002; 35:557-563. [PubMed: 12394384]

32. Perrin JM, Kuhlthau K, Chughtai A, Romm D, Kirschner BS, Ferry GD, et al. Measuring quality of life in pediatric patients with inflammatory bowel disease: psychometric and clinical characteristics. J Pediatr Gastroenterol Nutr. 2008; 46:164-171. [PubMed: 18223375]

33. Loonen HJ, Grootenhuis MA, Last BF, De Haan RJ, Bouquet J, Derkx BHF. Measuring quality of life in children with inflammatory bowel disease: the Impact-II (NL). Qual Life Res. 2002; 11:4756. [PubMed: 12003055]

34. Hood KK, Butler DA, Anderson BJ, Laffel LM. Updated and revised diabetes family conflict scale. Diabetes Care. 2007; 30:1764-1769. [PubMed: 17372149]

35. Janicke DM, Mitchell MJ, Stark LJ. Family functioning in school-age children with cystic fibrosis: an observational assessment of family interactions in the mealtime environment. J Pediatr Psychol. 2005; 30:179-186. [PubMed: 15681312]

36. Mackner LM, Crandall WV. Long-term psychosocial outcomes reported by children and adolescents with inflammatory bowel disease. Am J Gastroenterol. 2005; 100:1386-1392. [PubMed: 15929775]

37. Kazak AE. A contextual family/systems approach to pediatric psychology: introduction to the special issue. J Pediatr Psychol. 1997; 22:141-148. [PubMed: 9114639]

38. Wysocki T, Harris MA, Buckloh LM, Mertlich D, Lochrie AS, Taylor A, et al. Randomized, controlled trial of behavioral family systems therapy for diabetes: maintenance and generalization of effects on parent-adolescent communication. Behav Ther. 2008; 39:33-46. [PubMed: 18328868] 
Table 1

Participant characteristics $(N=62)$

\begin{tabular}{|c|c|c|}
\hline & $\%$ & Means \pm SD \\
\hline Adolescent age (years) & & $15.47 \pm 1.41$ \\
\hline Sex (\% female) & 43.5 & \\
\hline Ethnicity (\% white, not hispanic origin) & 88.7 & \\
\hline Primary caregiver marital status (\% married) & 85.5 & \\
\hline Primary caregiver education level ( $\%$ with at least a college degree) & 35.5 & \\
\hline Primary caregiver employment status (\% employed) & 80.7 & \\
\hline \multicolumn{3}{|l|}{ IBD diagnosis $(\%)$} \\
\hline Crohn's disease & 79.0 & \\
\hline Ulcerative colitis & 21.0 & \\
\hline \multicolumn{3}{|l|}{ Disease severity } \\
\hline PCDAI & & $11.68 \pm 10.01$ \\
\hline Inactive disease & 40.8 & \\
\hline Mild disease & 51.0 & \\
\hline Moderate-to-severe disease & 8.2 & \\
\hline LCAI & & $2.85 \pm 3.87$ \\
\hline Quiescent disease & 61.5 & \\
\hline Response to therapy & 30.8 & \\
\hline Active disease/no response to therapy & 7.7 & \\
\hline FAD problem solving & & $1.84 \pm 0.37$ \\
\hline FAD communication & & $1.93 \pm 0.42$ \\
\hline FAD roles & & $2.14 \pm 0.33$ \\
\hline FAD affective responsiveness & & $1.76 \pm 0.48$ \\
\hline FAD affective involvement & & $1.86 \pm 0.42$ \\
\hline FAD behavior control & & $1.54 \pm 0.32$ \\
\hline FAD general functioning & & $1.66 \pm 0.37$ \\
\hline IMPACT-III general well-being & & $58.03 \pm 8.74$ \\
\hline IMPACT-III emotional functioning & & $46.13 \pm 7.12$ \\
\hline IMPACT-III social functioning & & $21.87 \pm 2.77$ \\
\hline IMPACT-III body image & & $11.32 \pm 2.41$ \\
\hline IMPACT-III total HRQOL & & $144.52 \pm 19.91$ \\
\hline
\end{tabular}

FAD, family assessment device; HRQOL, health-related quality of life; IBD, inflammatory bowel disease; LCAI, Lichtiger colitis activity index; PCDAI, pediatric Crohn's disease activity index; SD, standard deviation. 